\title{
Exosomes from mesenchymal stem/stromal cells: a new therapeutic paradigm
}

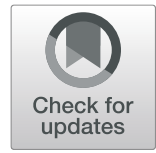

Kan Yin ${ }^{2}$, Shihua Wang ${ }^{1 *}$ and Robert Chunhua Zhao ${ }^{1 *}$

\begin{abstract}
Mesenchymal stem/stromal cells (MSCs) have been demonstrated to hold great potential for the treatment of several diseases. Their therapeutic effects are largely mediated by paracrine factors including exosomes, which are nanometer-sized membrane-bound vesicles with functions as mediators of cell-cell communication. MSCderived exosomes contain cytokines and growth factors, signaling lipids, mRNAs, and regulatory miRNAs. Increasing evidence suggests that MSC-derived exosomes might represent a novel cell-free therapy with compelling advantages over parent MSCs such as no risk of tumor formation and lower immunogenicity. This paper reviews the characteristics of MSC exosomes and their fate after in vivo administration, and highlights the therapeutic potential of MSC-derived exosomes in liver, kidney, cardiovascular and neurological disease. Particularly, we summarize the recent clinical trials performed to evaluate the safety and efficacy of MSC exosomes. Overall, this paper provides a general overview of MSCexosomes as a new cell-free therapeutic paradigm.
\end{abstract}

Keywords: Exosomes, Mesenchymal stem cell, Clinical trial, Disease

\section{Background}

Mesenchymal stem/stromal cells (MSCs) are one of the most commonly employed cell types as a cell-based therapy for treating human diseases. Recently, several mechanisms have been put forward regarding the therapeutic potential of MSCs, including (1) paracrine factors involving proteins/peptides and hormones and (2) the transfer of exosomes/microvesicles packaging various molecules [1]. The therapeutic potential of mesenchymal stromal cells (MSCs) may be largely mediated by paracrine factors contained in vesicles [2]. Extracellular vesicles (EVs) from many cell sources have now been recognized as important messengers in intercellular communication via transfer of bioactive lipids, proteins, and RNAs. EVs are generally divided into 3 subgroups depending on their biogenesis; (a) exosomes, with a diameter of $40-150 \mathrm{~nm}$, which are released into the extracellular when multivesicular bodies fuse with the cell membrane, (b) microvesicles, with a diameter of $150-1000 \mathrm{~nm}$, originating from direct budding of the plasma membrane and finally (c) apoptotic bodies, which display a broad size distribution (50-2000 nm) [3]. Exosomes are crucial messengers that present in biological fluids and are involved in multiple physiological and pathological processes [4]. Today, there are hundreds of clinics and hundreds of clinical trials using human MSCs with very few, if any, focusing on the in vitro multipotential capacities of these cells, these cells home in on sites of injury or disease and secrete bioactive factors that are immunomodulatory and trophic (regenerative) [5]. One advantage of using exosomes is to get around MSCs' side effects, exosomes are nanoparticles that can penetrate blood brain barrier and avoid potential pulmonary embolism related to transplantation of MSCs [6]. Knowledge of exosomes is essential to shed light on the functions of these vesicles on clinical applications. In this review, we focus on the mechanisms of exosomes covering the current knowledge on their potential cell-free therapeutic applications for MSC-derived exosomes.

\section{Exosomes}

Exosomes are a family of nanoparticles with a diameter in the range of $40-150 \mathrm{~nm}$ that are generated inside multivesicular bodies (MVBs) and are secreted when

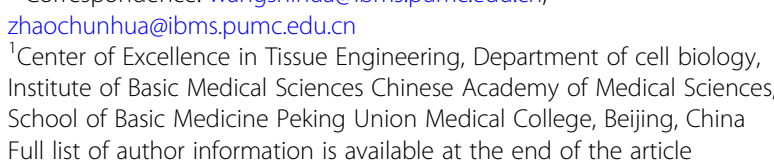

(c) The Author(s). 2019 Open Access This article is distributed under the terms of the Creative Commons Attribution 4.0 International License (http://creativecommons.org/licenses/by/4.0/), which permits unrestricted use, distribution, and reproduction in any medium, provided you give appropriate credit to the original author(s) and the source, provide a link to the Creative Commons license, and indicate if changes were made. The Creative Commons Public Domain Dedication waiver (http://creativecommons.org/publicdomain/zero/1.0/) applies to the data made available in this article, unless otherwise stated. 
these compartments fuse with the plasma membrane [7]. Upon the fusion of MVBs with the plasma membrane, exosomes are released into the extracellular and can be either taken up by target cells residing in the microenvironment or carried to distant sites via biological fluids [8]. Exosomes are enriched in many bioactive molecules such as lipids, proteins, mRNAs, transfer RNA (tRNA), long noncoding RNAs (lncRNAs), microRNAs (miRNAs) and mitochondrial DNA (mtDNA) [9]. Most exosomes have an evolutionarily conserved set of proteins including tetraspanins (CD81, CD63, and CD9), heat-shock proteins (HSP60, HSP70 and HSP90), ALIX and tumor susceptibility gene 101 (TSG101); however, they also have unique tissue type-specific proteins that reflect their cellular sources [10]. It has been reported that exosomes may be released from multiple cell types, including immunocytes [11], tumor cells [12], and mesenchymal stem/stromal cells (MSCs) [13]. Exosomes have received the most attention and have been implicated in physiological functions and in pathological conditions. Exosomes released by malignant cells play an important role in cancer cell communication with their microenvironment. HCC cell HepG2-derived exosomes could be actively internalized by adipocytes and caused significant transcriptomic alterations and in particular induced an inflammatory phenotype in adipocytes [14]. Exosomal miRNAs can affect many aspects of physiological and pathological conditions in $\mathrm{HCC}$ and indicates that miRNAs in exosomes can not only serve as sensitive biomarkers for cancer diagnostics and recurrence but can also potentially be used as therapeutics to target HCC progression [15].

\section{Characteristics of MSC-derived exosomes}

The abundance of cargos identified from MSC-derived exosomes function largely via the constant transfer of miRNAs and proteins, $>150$ miRNAs [16] and $>850$ unique protein [17] have been identified in the cargo of MSC-derived exosomes, resulting in the alteration of a variety of activities in target cells via different pathways. Many miRNAs have been found in MSC-derived exosomes and are reportedly involved in both physiological and pathological processes such as organism development, epigenetic regulation, immunoregulation (miR-155 and miR-146) [18], tumorigenesis and tumor progression (miR-23b, miR-451, miR-223, miR-24, miR-125b, miR-31, miR-214, and miR-122) [19]. Over 900 species of proteins have been collected from MSC-derived exosomes according to ExoCarta. Several studies have also shown that exosomes derived from MSCs harbor cytokines and growth factors, such as TGF $\beta 1$, interleukin-6 (IL-6), IL-10, and hepatocyte growth factor (HGF), which have been proven to contribute to immunoregulation [20]. Comparable levels of VEGF, extracellular matrix metalloproteinase inducer (EMMPRIN), and MMP-9 have been reported in MSC-derived exosomes, these three proteins play a vital role in stimulating angiogenesis, which could be fundamental for tissue repair [21].

\section{The fate of injected MSC-derived exosomes}

Current knowledge of the biodistribution of EVs upon administration in animal models is limited. Do MSC-derived exosomes have a favorable biodistribution and pharmacokinetic profile? Several strategies have been employed for in vivo tracking to determine EVs biodistribution upon systemic delivery in different animal models [22, 23]. Near-infrared (NIR) dyes are ideal for in vivo applications due to their high signal/noise ratio [24]. EVs with superparamagnetic iron oxide nanoparticles for high resolution and sensitive magnetic resonance analysis provide for accurate detection also in deep organs [25]. In an intracerebral hemorrhage rat model, DiI-labeled MSC-derived exosomes reached brain, liver, lung, and spleen after intravenous injection [26]. Exosomes appear to be able to home to the injury site. In the mouse model of acute kidney injury (AKI), DiD-labeled EVs were accumulated specifically in the kidneys of mice with AKI compared with healthy controls [27]. Intranasal administration led to better brain accumulation of exosomes at the injured brain site, compared to i.v. injection [28]. Biodistribution of systemically administered EVs is a dynamic process: a rapid phase of distribution in liver, spleen, and lungs within approximately $30 \mathrm{~min}$ upon administration is followed by an elimination phase via hepatic and renal processing, removing EVs in 1 to $6 \mathrm{~h}$ after administration [29].

\section{Therapeutic effects of MSC-derived exosomes Liver diseases}

The application of MSCs in animal models of liver fibrosis/cirrhosis and acute liver injury, eventually, in patients ameliorates the progress of the disease. $\mathrm{Li}$ et al. found that the exosomes derived from human umbilical cord MSCs (hucMSC) ameliorate liver fibrosis by inhibiting both the epithelial-mesenchymal transition of hepatocytes and collagen production, significantly restore the serum aspartate aminotransferase activity and inactivate the TGF- $\beta 1 /$ Smad 2 signaling pathway by decreasing collagen type I/III and TGF- $\beta 1$ and the phosphorylation of Smad2 [30]. Tan et al. found that HuES9.E1 MSC-derived exosomes elicit hepatoprotective effects through an increase in hepatocyte proliferation, as demonstrated by high expression of proliferation proteins (proliferating cell nuclear antigen and Cyclin D1), the anti-apoptotic gene $\mathrm{Bcl}-\mathrm{xL}$ and the signal transducer and activator of transcription 3 (STAT3) [31]. Liver regeneration was significantly stimulated by MSCs culture medium (MSC-CM) as shown by an increase in liver to 
body weight ratio and hepatocyte proliferation. MSC-CM upregulated hepatic gene expression of cytokines and growth factors relevant for cell proliferation, angiogenesis, and anti-inflammatory responses, treatment with MSC-derived factors can promote hepatocyte proliferation and regenerative responses in the early phase after surgical resection [32]. Transplantation of exosomes released from adipose derived-MSCs (AD-MSC) can significantly reduce the elevated serum levels of alanine aminotransferase and aspartate aminotransferase, liver inflammation and necrosis in concanavalin A (Con A)-induced hepatitis in C57BL/6 mice as well as the serum levels of proinflammatory cytokines, including tumor necrosis factor- $\alpha$ (TNF- $\alpha$ ), interferon- $\gamma$ (IFN- $\gamma$ ), IL-6, IL-18 and IL-1 $\beta$, and the inflammasome activation in mouse liver [33].

\section{Kidney disease}

Mesenchymal stem/stromal cells (MSCs) have shown promising results in experimental acute kidney injury (AKI) and chronic kidney disease (CKD). Systemic administration of human umbilical cord-derived MSCs (huMSCs)-derived EVs in rats with renal Ischemia-reperfusion injury (IRI) increased renal capillary density and reduced fibrosis by direct transfer of the proangiogenic factor vascular endothelial growth factor (VEGF) and mRNAs involved in this process [34]. A single intrarenal administration of adipose tissue-derived autologous MSCs-derived EVs in pigs with renal artery stenosis attenuated renal inflammation, disclosed by decreased renal vein levels of several pro-inflammatory cytokines, including TNF- $\alpha$, IL- 6 , and IL-1- $\beta$. Contrarily, renal vein levels of IL-10 increased in EV-treated pigs, associated with a shift from pro-inflammatory to reparative macrophages populating the stenotic kidney, underscoring the immunomodulatory potential of EVs [35]. Microvesicles derived from human bone marrow MSCs stimulated proliferation in vitro and conferred resistance of tubular epithelial cells to apoptosis. In vivo, microvesicles accelerated the morphologic and functional recovery of glycerol-induced acute kidney injury (AKI) in SCID mice by inducing proliferation of tubular cells. Microarray analysis and quantitative real time PCR of microvesicle-RNA extracts indicate that microvesicles shuttle a specific subset of cellular mRNA, such as mRNAs associated with the mesenchymal phenotype and with control of transcription, proliferation, and immunoregulation [36]. The effects of bone marrow MSCs-derived MVs in SCID mice survival in lethal cisplatin-induced acute renal injury (AKI) was to exert a pro-survival effect on renal cells in vitro and in vivo mainly ascribed to an anti-apoptotic effect of MVs. MVs up-regulated in cisplatin-treated human tubular epithelial cells anti-apoptotic genes, such as Bcl-xL, Bcl2 and
BIRC8 and down-regulated genes that have a central role in the execution-phase of cell apoptosis such as Casp1, Casp8 and LTA [37]. Intravenous injection of EVs isolated from the conditioned medium of human umbilical cord MSCs after unilateral renal ischemia preserved kidney function and decreased serum levels of the AKI marker neutrophil gelatinase-associated lipocalin [38]. Human bone marrow MSCs-derived exosomes contain insulin-like growth factor-1 receptor (IGF-1R) mRNA. Exosomal transfer of IGF-1R mRNA to damaged renal tubular cells promoted their proliferation and repair and this effect was significantly reduced when IGF-1R transcription in donor cells was silenced [39].

\section{Cardiovascular disease}

There are preclinical studies in which MSC-derived exosomes are used for treating cardiovascular diseases (CVDs) such as AMI, stroke, pulmonary hypertension, and septic cardiomyopathy [40]. Cui et al. demonstrated adipose-derived MSC (AdMSC)-derived exosomes led to a markedly increase in cell viability of $\mathrm{H} 9 \mathrm{C} 2$ cells under hypoxia/reoxygenation $(\mathrm{H} / \mathrm{R})$ in vitro, and administration of AdMSC-derived exosomes protected ischemic myocardium from myocardial ischemia-reperfusion $(\mathrm{MI} / \mathrm{R})$ injury via activation of Wnt/ $\beta$-catenin signaling in vivo [41]. Furthermore, Wang et al. showed superior cardioprotective effects of endometrium-derived MSCs (EmMSC) in a rat myocardial infarction (MI) model as compared to BMSCs and AdMSCs. These differences may be caused by certain miRNAs particularly miR-21 enrichment in exosomes secreted from EmMSCs, which exerted effects on cell survival and angiogenesis by targeting PTEN [42]. HuES9.E1 derived MSCs-derived exosomes treatment increased levels of ATP and $\mathrm{NADH}$, decreased oxidative stress, increased phosphorylated-Akt and phosphorylated-GSK-3 $\beta$, reduced phosphorylated-c-JNK in ischemic/reperfused hearts to enhance myocardial viability and prevented adverse remodeling after myocardial ischemia/reperfusion injury [43]. Feng et al. determined that miR-22 is highly enriched in exosomes secreted by mouse bone marrow-derived MSCs after ischemic preconditioning, and administration of these exosomes significantly reduced infarct size and cardiac fibrosis by targeting methyl-CpG-binding protein 2 (Mecp2) in a mouse myocardial infarction (MI) model [44]. Both bone marrow MSCs and their derived exosomes are cardioprotective against myocardial infarction in animal models. However, anti-miR-125b treatment of exosomes significantly attenuated their protective effect [45]. MiR-21-5p plays a key role in hMSC-exo-mediated effects on cardiac contractility and calcium handling, likely via PI3K signaling [46]. In a rat myocardial 
ischaemia reperfusion injury model, injection of bone marrow-derived MSCs-derived exosomes reduced apoptosis and myocardial infarct size and subsequently improved heart functions by inducing cardiomyocyte autophagy via AMPK/mTOR and Akt/mTOR pathways [47].

\section{Neurological disease}

MSC-Exosomes have shown potential therapeutic benefit in the treatment of neurological and neurodegenerative diseases. One of the most outstanding results in the field is the fact that systemically injected exosomes are able to cross the blood-brain barrier (BBB) and achieve the brain parenchyma. Systemic delivery of targeted exosomes containing a siRNA against $\alpha$-synuclein reduced the mRNA and protein levels of $\alpha$-synuclein in the brain [48, 49]. Xin et al. also reported that rat bone marrow derived MSCs derived EVs enriched with the miR-17-92 cluster enhanced oligodendrogenesis neurogenesis neural plasticity and functional recovery after stroke possibly by suppressing PTEN and subsequently by increasing the phosphorylation of proteins downstream of PTEN including of the protein kinase $\mathrm{B} /$ mechanistic target of rapamycin/glycogen synthase kinase $3 \beta$ signaling pathway [50]. Katsuda et al. used exosomes secreted from human adipose tissue-derived MSCs that contain large amounts of neprilysin, the most prominent enzyme that degrades $\beta$-amyloid peptide in the brain. Transfer of exosomes into neuroblastoma N2a cells led to reductions in both secreted and intracellular $\beta$-amyloid peptide levels, which might be a therapeutic approach to Alzheimer's disease [51]. The results of migration assay and capillary network formation assay showed that exosomes secreted by adipose-derived stem cells (ADSCs-Exos) promoted the mobility and angiogenesis of brain microvascular endothelial cells (BMECs) after oxygen-glucose deprivation (OGD) via miR-181b-5p/TRPM7 axis [52]. Injection of exosomes from mouse bone marrow MSCs could rescue cognition and memory impairment according to results of the Morris water maze test, reduced plaque deposition, and $A \beta$ levels in the brain; could decrease the activation of astrocytes and microglia; could down-regulate proinflammatory cytokines (TNF- $\alpha$ and IL-1 $\beta$ ); and could up-regulate anti-inflammatory cytokines (IL-4 and -10) in AD mice, as well as reduce the activation of signal transducer and activator of transcription 3 (STAT3) and $\mathrm{NF}-\kappa \mathrm{B}$ in APP/PS1 double transgenic mice [53].

Immune disease Potent immunomodulatory properties of MSCs-exo has been evaluated. Exosomes have been observed to play crucial roles in carrying and presenting

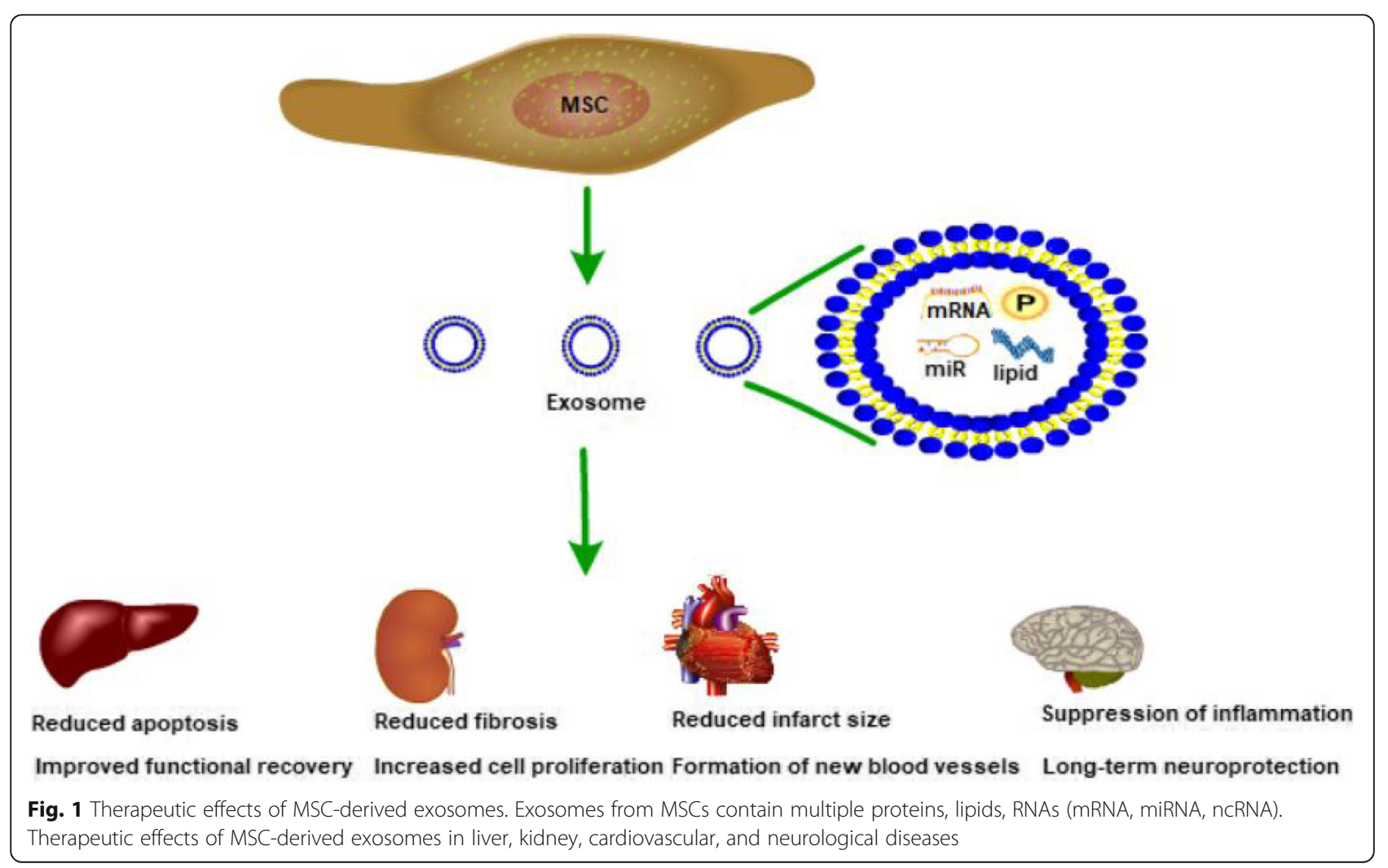


functional MHC-peptide complexes to modulate tumor-specific T cell activation [54]. Exosomes released from Bone marrow (BM)-derived MSCs can effectively ameliorate chronic graft-versus-host disease (cGVHD) in mice by inhibiting the activation and infiltration of CD4 $\mathrm{T}$ cells, reducing pro-inflammatory cytokine production, as well as improving the generation of IL-10-expressing Treg and inhibiting Th17 cells [55]. Human multipotent stromal cells-derived EVs suppress autoimmunity in models of type 1 diabetes (T1D) and experimental autoimmune uveoretinitis (EAU). EVs inhibit activation of antigen-presenting cells and suppress development of $\mathrm{T}$ helper 1 (Th1) and Th17 cells, they also increased expression of the immunosuppressive cytokine IL-10 and suppressed Th17 cell development [56]. Human bone-marrow derived MSCs exosomes promote Tregs proliferation and immunosuppression capacity by upregulating suppressive cytokines IL-10 and TGF- $\beta 1$ in PBMCs of asthmatic patient [57]. MiR-181c in human umbilical cord MSCs-derived exosomes is key to anti-inflammatory effects in burned rat inflammation model by downregulating the TLR4 signaling pathway [58] Fig. 1.

\section{Clinical trials of MSCs exosomes-based therapies}

The use of MSC-derived EVs for regenerative therapy requires the production and isolation of a suitable quantity of clinical grade EVs from cultured MSCs [59]. While complexities surrounding the therapeutic potential of MSCs exosomes continue to unravel, several clinical trials (Table 1, data from http://clinicaltrials.gov) have been completed or are underway in order to evaluate this therapeutic potential. Among them, determining the optimal dose, the appropriate time window for exosome administration and route of administration that achieves maximal efficacy without adverse effects are the most important issues to resolve [60]. Improved preclinical study quality in terms of treatment allocation reporting, randomization and blinding will accelerate needed progress towards clinical trials that should assess the feasibility and safety of this therapeutic approach in humans [61]. For example, MSC-exosomes will be great

Table 1 The function of MSC-derived exosomes

\begin{tabular}{|c|c|c|c|}
\hline Source of Exosomes & Specific Disease Treated & Target/Pathway & Reference \\
\hline human umbilical cord MSCs & liver fibrosis & TGF- $\beta 1 /$ Smad2 & [30] \\
\hline HuES9.E1 MSC & hepatoprotective effects & Cyclin D1, Bcl-xL, STAT3 & [31] \\
\hline adipose derived-MSCs & hepatitis & TNF- $a$, IFN- $\gamma$, IL-6, IL-18 and IL-1 $\beta$ & [33] \\
\hline human umbilical cord-derived MSCs & renal Ischemia-reperfusion injury (IRI) & VEGF & [34] \\
\hline adipose tissue-derived autologous MSCs & renal artery stenosis & TNF- $\alpha$, IL-6, IL10 and IL-1- $\beta$ & [35] \\
\hline human bone marrow MSCs & acute kidney injury & mRNAs & [36] \\
\hline bone marrow MSCs & acute renal injury & BCl-xL,BCl2, BIRC8,Casp1, Casp8 and LTA & [37] \\
\hline human umbilical cord MSCs & unilateral renal ischemia & lipocalin & [38] \\
\hline bone marrow MSCs & acute kidney injury & mRNAs & [36] \\
\hline Human bone marrow MSCs & damaged renal tubular & IGF-1R & [39] \\
\hline adipose-derived MSC & myocardial ischemia-reperfusion injury & Wnt/ß-catenin & [41] \\
\hline endometrium-derived MSCs & myocardial infarction & miR-21, PTEN & [42] \\
\hline HuES9.E1 derived MSCs & myocardial ischemia/reperfusion injury & PI3K/Akt & [43] \\
\hline mouse bone marrow-derived MSCs & myocardial infarction & miR-22, Mecp2 & [44] \\
\hline bone marrow MSCs & myocardial infarction & miR-125b & [45] \\
\hline human mesenchymal stem cell & cardiac contractility & miR-21-5p, PI3K & [46] \\
\hline bone marrow-derived MSCs & myocardial ischaemia reperfusion injury & AMPK/mTOR, Akt/mTOR & [47] \\
\hline rat bone marrow derived MSCs & stroke & miR-17-92, PTEN & [50] \\
\hline human adipose tissue-derived MSCs & Alzheimer's disease & neprilysin & [51] \\
\hline adipose-derived stem cells & oxygen-glucose deprivation & MicroRNA-181b/TRPM7 & [52] \\
\hline mouse bone marrow MSCs & Alzheimer's disease & STAT3, NF-KB & [53] \\
\hline bone marrow derived MSCs & chronic graft-versus-host disease & Treg, Th17 & [55] \\
\hline human multipotent stromal cells & type 1 diabetes, uveoretinitis & Th1, Th17 & [56] \\
\hline human bone-marrow derived MSCs & asthma & IL-10, TGF- $\beta 1$ & [57] \\
\hline human umbilical cord MSCs & inflammation & MiR-181C, TLR4 & [58] \\
\hline
\end{tabular}


Table 2 Clinical trials of MSCs exosomes-based therapies

\begin{tabular}{|c|c|c|c|c|}
\hline Study title & Disease & Intervention & Phase & NCT \\
\hline $\begin{array}{l}\text { Allogenic Mesenchymal Stem Cell-Derived Exosome in } \\
\text { Patients With Acute Ischemic Stroke }\end{array}$ & $\begin{array}{l}\text { Cerebrovascular } \\
\text { Disorders }\end{array}$ & Biological: exosome & $\begin{array}{l}\text { Phase } 1 \\
\text { Phase } 2\end{array}$ & NCT03384433 \\
\hline MSC-Exos Promote Healing of MHs & Macular Holes & $\begin{array}{l}\text { Biological: exosomes derived from } \\
\text { mesenchymal stem cells (MSC-Exo) }\end{array}$ & $\begin{array}{l}\text { Early } \\
\text { Phase } 1\end{array}$ & NCT03437759 \\
\hline microRNAs Role in Pre-eclampsia Diagnosis & Preeclampsia & Biological: exosome & Complete & NCT03562715 \\
\hline $\begin{array}{l}\text { Effect of Microvesicles and Exosomes Therapy on } \beta \text {-cell } \\
\text { Mass in Type I Diabetes Mellitus (T1DM) }\end{array}$ & $\begin{array}{l}\text { Diabetes Mellitus } \\
\text { Type } 1\end{array}$ & Biological: MSC exosomes. & $\begin{array}{l}\text { Phase } 2 \\
\text { Phase } 3\end{array}$ & NCT02138331 \\
\hline $\begin{array}{l}\text { Trial of a Vaccination With Tumor Antigen-loaded } \\
\text { Dendritic Cell-derived Exosomes }\end{array}$ & $\begin{array}{l}\text { Non Small Cell } \\
\text { Lung Cancer }\end{array}$ & Biological: Dex2 & Phase 2 & NCT01159288 \\
\hline $\begin{array}{l}\text { Serum Exosomal Long Noncoding RNAs as Biomarkers } \\
\text { for Lung Cancer Diagnosis }\end{array}$ & $\begin{array}{l}\text { Lung Cancer } \\
\text { (Diagnosis) }\end{array}$ & Diagnostic Test: collect samples & & NCT03830619 \\
\hline
\end{tabular}

biological tools for cancer therapy, it is hopeful to delve deeper into the potential of MSC-exosomes among cancer cells and provide effective treatments with the highest safety [62] Table 2.

\section{Conclusions}

MSCs most exert their therapeutic effects through the secretion of factors to reduce cellular injury and enhance repair. MSC exosomes probably function in a similar fashion, namely as a communication vehicle secreted by MSCs to affect the stromal support functions through the maintenance of a dynamic and homeostatic tissue microenvironment [63]. MSC exosomes may have the versatility and capacity to interact with multiple cell types within the immediate vicinity and remote areas to elicit appropriate cellular responses. MSCs through their secreted exosomes target housekeeping processes to restore tissue homeostasis and enable cells within the tissue to recover, repair and regenerate. This hypothesis provides a rationale for the therapeutic efficacy of MSCs and their secreted exosomes in a wide spectrum of diseases and rationalizes the additional use of MSC exosomes as an adjuvant to support and complement other therapeutic modalities [64]. Nonetheless, the exact mechanism of in vivo action of exogenously administered exosomes, their biodistribution, pharmacokinetics, and possibility of targeted delivery are not fully elucidated. New techniques may help in filling this gap of knowledge and further promoting clinical translation of exosomes-based regenerative therapy [65].

\footnotetext{
Abbreviations

AKI: Acute kidney injury; BBB: Blood-brain barrier; CKD: Chronic kidney disease; EVs: Extracellular vesicles; H/R: Hypoxia/reoxygenation; MI/ R: Myocardial ischemia-reperfusion; MSCs: Mesenchymal stem/stromal cells; MVBs: multivesicular bodies; NIR: Near-infrared; OGD: Oxygen-glucose deprivation
}

\section{Acknowledgments}

The author thanks Shihuan Wang, PhD, for critical reading of the manuscript and numerous helpful discussions.

\section{Funding}

For funding, we acknowledge the study was supported by CAMS Innovation Fund for Medical Sciences (2017-12M-3-007), Beijing Key Laboratory of New Drug Development and Clinical Trial of Stem Cell Therapy (BZ0381), Qingdao University newly introduced talent research start-up fee (41117010251).

\section{Availability of data and materials}

Data sharing is not applicable to this article as no datasets were generated or analyzed during the current study.

\section{Authors' contributions \\ KY drafted the manuscript. RCZ and SW supervised and revised the manuscript. All authors read and approved the final manuscript.}

Ethics approval and consent to participate

Not applicable.

\section{Consent for publication}

Not applicable.

\section{Competing interests}

The authors declare that they have no competing interests.

\section{Publisher's Note}

Springer Nature remains neutral with regard to jurisdictional claims in published maps and institutional affiliations.

\section{Author details}

${ }^{1}$ Center of Excellence in Tissue Engineering, Department of cell biology, Institute of Basic Medical Sciences Chinese Academy of Medical Sciences, School of Basic Medicine Peking Union Medical College, Beijing, China. ${ }^{2}$ Department of Biochemistry and Molecular biology, Basic medical college, Qingdao University, 308 Ningxia Road, Qingdao 266071, China.

Received: 29 January 2019 Accepted: 27 March 2019

Published online: 04 April 2019

\section{References}

1. Spees JL, Lee RH, Gregory CA. Mechanisms of mesenchymal stem/stromal cell function. Stem Cell Res Ther. 2016;7(1):125.

2. Akyurekli $\mathrm{C}$, et al. A systematic review of preclinical studies on the therapeutic potential of mesenchymal stromal cell-derived microvesicles. Stem Cell Rev. 2015;11(1):150-60.

3. El Andaloussi S, et al. Extracellular vesicles: biology and emerging therapeutic opportunities. Nat Rev Drug Discov. 2013;12(5):347-57.

4. Yeo RW, et al. Mesenchymal stem cell: an efficient mass producer of exosomes for drug delivery. Adv Drug Deliv Rev. 2013;65(3):336-41.

5. Caplan Al. Mesenchymal stem cells: time to change the name! Stem Cells Transl Med. 2017;6(6):1445-51.

6. Jung JW, et al. Familial occurrence of pulmonary embolism after intravenous, adipose tissue-derived stem cell therapy. Yonsei Med J. 2013;54(5):1293-6. 
7. Tkach $M$, Thery $C$. Communication by extracellular vesicles: where we are and where we need to go. Cell. 2016;164(6):1226-32.

8. Hyenne $\mathrm{V}$, et al. RAL-1 controls multivesicular body biogenesis and exosome secretion. J Cell Biol. 2015;211(1):27-37.

9. Keerthikumar $\mathrm{S}$, et al. ExoCarta: a web-based compendium of Exosomal cargo. J Mol Biol. 2016:428(4):688-92.

10. Schey KL, Luther JM, Rose KL. Proteomics characterization of exosome cargo. Methods. 2015;87:75-82.

11. Shen M, Ren X. New insights into the biological impacts of immune cellderived exosomes within the tumor environment. Cancer Lett. 2018;431: $115-22$.

12. Gao $\mathrm{L}$, et al. Tumor-derived exosomes antagonize innate antiviral immunity. Nat Immunol. 2018;19(3):233-45.

13. Cosenza S, et al. Mesenchymal stem cells-derived exosomes are more immunosuppressive than microparticles in inflammatory arthritis. Theranostics. 2018;8(5):1399-410.

14. Wang $\mathrm{S}$, et al. Exosomes released by hepatocarcinoma cells endow adipocytes with tumor-promoting properties. J Hematol Oncol. 2018; 11(1):82.

15. Li S, et al. Exosomal miRNAs in hepatocellular carcinoma development and clinical responses. J Hematol Oncol. 2018:11(1):54.

16. Chen TS, et al. Mesenchymal stem cell secretes microparticles enriched in pre-microRNAs. Nucleic Acids Res. 2010;38(1):215-24.

17. Lai RC, et al. Proteolytic potential of the MSC exosome proteome: implications for an exosome-mediated delivery of therapeutic proteasome. Int J Proteomics. 2012;2012:971907.

18. Di Trapani $\mathrm{M}$, et al. Differential and transferable modulatory effects of mesenchymal stromal cell-derived extracellular vesicles on T, B and NK cell functions. Sci Rep. 2016;6:24120.

19. Ono $\mathrm{M}$, et al. Exosomes from bone marrow mesenchymal stem cells contain a microRNA that promotes dormancy in metastatic breast cancer cells. Sci Signal. 2014;7(332):ra63.

20. Burrello J, et al. Stem cell-derived extracellular vesicles and immunemodulation. Front Cell Dev Biol. 2016:4:83.

21. Vrijsen KR, et al. Exosomes from cardiomyocyte progenitor cells and mesenchymal stem cells stimulate angiogenesis via EMMPRIN. Adv Healthc Mater. 2016;5(19):2555-65.

22. Wiklander $\mathrm{OP}$, et al. Extracellular vesicle in vivo biodistribution is determined by cell source, route of administration and targeting. J Extracell Vesicles. 2015:4:26316.

23. Choi H, Lee DS. Illuminating the physiology of extracellular vesicles. Stem Cell Res Ther. 2016;7(1):55.

24. Tian T, et al. Visualizing of the cellular uptake and intracellular trafficking of exosomes by live-cell microscopy. J Cell Biochem. 2010;111(2):488-96.

25. Busato $A$, et al. Labeling and magnetic resonance imaging of exosomes isolated from adipose stem cells. Curr Protoc Cell Biol. 2017;75:3.44.1-3.44.15.

26. Otero-Ortega $L$, et al. Exosomes promote restoration after an experimental animal model of intracerebral hemorrhage. J Cereb Blood Flow Metab. 2018:38(5):767-79.

27. Grange $C$, et al. Biodistribution of mesenchymal stem cell-derived extracellular vesicles in a model of acute kidney injury monitored by optical imaging. Int J Mol Med. 2014;33(5):1055-63.

28. Betzer $\mathrm{O}$, et al. In vivo neuroimaging of exosomes using gold nanoparticles. ACS Nano. 2017;11(11):10883-93.

29. Lai CP, et al. Dynamic biodistribution of extracellular vesicles in vivo using a multimodal imaging reporter. ACS Nano. 2014;8(1):483-94.

30. Li T, et al. Exosomes derived from human umbilical cord mesenchymal stem cells alleviate liver fibrosis. Stem Cells Dev. 2013;22(6):845-54.

31. Tan CY, et al. Mesenchymal stem cell-derived exosomes promote hepatic regeneration in drug-induced liver injury models. Stem Cell Res Ther. 2014; 5(3):76.

32. Fouraschen SM, et al. Secreted factors of human liver-derived mesenchymal stem cells promote liver regeneration early after partial hepatectomy. Stem Cells Dev. 2012:21(13):2410-9.

33. Lou G, et al. Mesenchymal stem cell-derived exosomes as a new therapeutic strategy for liver diseases. Exp Mol Med. 2017;49(6):e346.

34. Zou X, et al. Human mesenchymal stromal cell-derived extracellular vesicles alleviate renal ischemic reperfusion injury and enhance angiogenesis in rats. Am J Transl Res. 2016;8(10):4289-99.

35. Eirin $\mathrm{A}$, et al. Mesenchymal stem cell-derived extracellular vesicles attenuate kidney inflammation. Kidney Int. 2017;92(1):114-24.
36. Bruno $\mathrm{S}$, et al. Mesenchymal stem cell-derived microvesicles protect against acute tubular injury. J Am Soc Nephrol. 2009;20(5):1053-67.

37. Bruno $S$, et al. Microvesicles derived from mesenchymal stem cells enhance survival in a lethal model of acute kidney injury. PLoS One. 2012;7(3):e33115.

38. Zhang G, et al. Mesenchymal stromal cell-derived extracellular vesicles protect against acute kidney injury through anti-oxidation by enhancing Nrf2/ARE activation in rats. Kidney Blood Press Res. 2016;41(2):119-28.

39. Tomasoni $\mathrm{S}$, et al. Transfer of growth factor receptor mRNA via exosomes unravels the regenerative effect of mesenchymal stem cells. Stem Cells Dev. 2013;22(5):772-80

40. Suzuki E, et al. Therapeutic effects of mesenchymal stem cell-derived exosomes in cardiovascular disease. Adv Exp Med Biol. 2017;998:179-85.

41. Cui $X$, et al. Exosomes from adipose-derived mesenchymal stem cells protect the myocardium against ischemia/reperfusion injury through Wnt/ beta-catenin signaling pathway. J Cardiovasc Pharmacol. 2017;70(4):225-31.

42. Wang $\mathrm{K}$, et al. Enhanced Cardioprotection by human endometrium mesenchymal stem cells driven by Exosomal MicroRNA-21. Stem Cells Transl Med. 2017:6(1):209-22.

43. Arslan F, et al. Mesenchymal stem cell-derived exosomes increase ATP levels, decrease oxidative stress and activate PI3K/Akt pathway to enhance myocardial viability and prevent adverse remodeling after myocardial ischemia/reperfusion injury. Stem Cell Res. 2013;10(3):301-12.

44. Feng $Y$, et al. Ischemic preconditioning potentiates the protective effect of stem cells through secretion of exosomes by targeting Mecp2 via miR-22. PLoS One. 2014;9(2):e88685.

45. Xiao C, et al. Transplanted mesenchymal stem cells reduce Autophagic flux in infarcted hearts via the Exosomal transfer of miR-125b. Circ Res. 2018; 123(5):564-78.

46. Mayourian J, et al. Exosomal microRNA-21-5p mediates mesenchymal stem cell paracrine effects on human cardiac tissue contractility. Circ Res. 2018; 122(7):933-44.

47. Liu $L$, et al. Exosomes derived from mesenchymal stem cells rescue myocardial Ischaemia/reperfusion injury by inducing cardiomyocyte autophagy via AMPK and Akt pathways. Cell Physiol Biochem. 2017;43(1): $52-68$

48. Luarte A, et al. Potential therapies by stem cell-derived exosomes in CNS diseases: focusing on the neurogenic niche. Stem Cells Int. 2016:2016: 5736059.

49. Cooper JM, et al. Systemic exosomal siRNA delivery reduced alpha-synuclein aggregates in brains of transgenic mice. Mov Disord. 2014;29(12):1476-85.

50. Xin $\mathrm{H}$, et al. MicroRNA cluster miR-17-92 cluster in exosomes enhance neuroplasticity and functional recovery after stroke in rats. Stroke. 2017; 48(3):747-53.

51. Katsuda T, et al. Human adipose tissue-derived mesenchymal stem cells secrete functional neprilysin-bound exosomes. Sci Rep. 2013;3:1197.

52. Yang $Y$, et al. Exosomes secreted by adipose-derived stem cells contribute to angiogenesis of brain microvascular endothelial cells following oxygenglucose deprivation in vitro through MicroRNA-181b/TRPM7 Axis. J Mol Neurosci. 2018;65(1):74-83

53. Cui GH, et al. Exosomes derived from hypoxia-preconditioned mesenchymal stromal cells ameliorate cognitive decline by rescuing synaptic dysfunction and regulating inflammatory responses in APP/PS1 mice. FASEB J. 2018; 32(2):654-68.

54. You L, et al. The crosstalk between autophagic and endo-/exosomal pathways in antigen processing for MHC presentation in anticancer T cell immune responses. J Hematol Oncol. 2017;10(1):165.

55. Lai $\mathrm{P}$, et al. A potent immunomodulatory role of exosomes derived from mesenchymal stromal cells in preventing cGVHD. J Hematol Oncol. 2018; 11(1):135.

56. Shigemoto-Kuroda T, et al. MSC-derived extracellular vesicles attenuate immune responses in two autoimmune murine models: type 1 diabetes and Uveoretinitis. Stem Cell Reports. 2017;8(5):1214-25.

57. Du YM, et al. Mesenchymal stem cell exosomes promote immunosuppression of regulatory T cells in asthma. Exp Cell Res. 2018; 363(1):114-20.

58. Li X, et al. Exosome derived from human umbilical cord mesenchymal stem cell mediates MiR-181c attenuating burn-induced excessive inflammation. EBioMedicine. 2016;8:72-82.

59. Rani $\mathrm{S}$, et al Mesenchymal stem cell-derived extracellular vesicles: toward cell-free therapeutic applications. Mol Ther. 2015;23(5):812-23. 
60. Cheng $L$, et al. Focus on mesenchymal stem cell-derived exosomes: opportunities and challenges in cell-free therapy. Stem Cells Int. 2017;2017: 6305295.

61. Lavoie JR, Rosu-Myles M. Uncovering the secretes of mesenchymal stem cells. Biochimie. 2013;95(12):2212-21.

62. Zhou J, et al. Mesenchymal stem cell derived exosomes in Cancer progression, metastasis and drug delivery: a comprehensive review. J Cancer. 2018;9(17):3129-37.

63. Katsuda T, Ochiya T. Molecular signatures of mesenchymal stem cell-derived extracellular vesicle-mediated tissue repair. Stem Cell Res Ther. 2015;6:212.

64. Kupcova Skalnikova H. Proteomic techniques for characterisation of mesenchymal stem cell secretome. Biochimie. 2013;95(12):2196-211.

65. Morishita M, et al. Quantitative analysis of tissue distribution of the B16BL6derived exosomes using a streptavidin-lactadherin fusion protein and iodine-125-labeled biotin derivative after intravenous injection in mice. $J$ Pharm Sci. 2015;104(2):705-13.

Ready to submit your research? Choose BMC and benefit from:

- fast, convenient online submission

- thorough peer review by experienced researchers in your field

- rapid publication on acceptance

- support for research data, including large and complex data types

- gold Open Access which fosters wider collaboration and increased citations

- maximum visibility for your research: over $100 \mathrm{M}$ website views per year

At $\mathrm{BMC}$, research is always in progress.

Learn more biomedcentral.com/submissions 